\title{
User-driven innovation of an outpatient department
}

\author{
Ole Broberg* and Kasper Edwards \\ Department of Management Engineering, Technical University of Denmark, Building 424, DK-2800 Lyngby, \\ Denmark, +45 45256033
}

\begin{abstract}
This paper presents experiences from a user-driven innovation process of an outpatient department in a hospital. The mixing of methods from user-driven innovation and participatory design contributed to develop an innovative concept of the spatial and organizational design of an outpatient department in a hospital. Design games and tabletop simulation seem to be powerful tools in facilitating such a participatory process.
\end{abstract}

Keywords: innovation, user participation, codesign, health care sector, outpatient department

\section{Introduction}

The Danish healthcare sector is a public healthcare system where everyone is entitled to treatment. The future development indicates a healthcare with increasingly sophisticated care and significantly more outpatient activity. Thus the Danish hospital sector is facing a major renewal over the next ten years with the construction of several new so-called "super" hospitals.

In a parallel development, the Copenhagen area regional council is planning to set up a Healthcare Innovation Lab (HIL). This facility is aimed at being partner in innovation projects with companies in the private sector. The HIL will be offering facilities and competences in testing new ideas for the healthcare sector. A major approach will be full-scale simulation by the help of hospital staff. Testing may include new IT systems, work organization, equipment, layout of departments, and interior design.

In order to develop a business case for the future HIL, a two-year research and development program was launched. This paper reports findings from one case in the HIL program: A project on user-driven innovation of an outpatient department. The purpose of the project was to develop and test methods for organizational development in healthcare with a focus on simulation. As a case this project collaborated with an outpatient department with 426 employees and has approx. 18.000 patient visits a year. The department was facing a move to an entirely new building within five to six years. Consultants and researchers planned and completed a user-driven innovation process aiming at innovating and designing the future organizational and functional features of the department. The research question in this explorative study is: What can be learned from an interactive research program on user-driven innovation of an outpatient department?

The concept of user-driven innovation is not clearly defined. However, in a Danish context, it has been defined as a process "in which you collect knowledge from the users aiming at developing new products, services and concepts. A user-driven innovation process is based on insights in user needs and a systematic involvement of the users"[1] Within this framework two dimensions are distinguished: How the users are involved, and if the project goal is to identify the users' acknowledged needs or nonacknowledged needs. Some of the main methods for user-driven innovation are ethnography, participatory design/innovation, and lead-user method.

Participatory design (PD) has its roots in the 1970s in Scandinavia and was initiated by academics that cooperated with people from trade unions. In PD, users are treated as experts, and it is attempted to

\footnotetext{
*Corresponding author. E-mail: obro@man.dtu.dk.
} 
bring their (tacit) knowledge and skills into the research and design process [2]. In participatory innovation the focus is widened to include business processes as well. The point of departure is that the design in itself is not enough to create a successful innovation. The design of a business model is as equal importance. Participatory innovation builds on crossdisciplinary collaboration between users, design anthropologists, sociologists, industrial designers, interaction designers, engineers, economists and people skilled in marketing and innovation management. Codesign, sometimes termed co-creation, can be understood as an attempt to "facilitate users, researchers, designers and others to cooperate creatively, so that they can jointly explore and envision ideas, make and discuss sketches, and tinker with mock-ups or prototypes" [2]. Codesign focuses on developing and using 'generative tools' that can establish 'a shared design language' and enable people to 'communicate visually and directly with each other' during the creative process [2].

\section{Method}

This project was accomplished within an interactive research framework, stressing that both the practitioner and the research system would gain new knowledge and experience. The research team consisted of five people with engineering or nursing background. A user group from the outpatient department was formed and included two medical doctors, two nurses, and one secretary. The project included three main phases: 1) researchers' observations of current work practice in the outpatient department, 2) four codesign workshops with five staff members from the department aiming at designing the future department, and 3) three tabletop simulation workshops aimed at innovating the organization and functional layout of the future outpatient department.

\section{Results}

\subsection{Field observations}

The research team spent two days on ethnographic inspired observations and video recording in the outpatient department. Observation of current work practice identified a number of factors that lowered efficiency of the department, some of which being:
- The updating of medical records was far behind the inflow, resulting in a huge accumulation of records.

- Inadequate work processes in the reception resulting in a confusing mixture of patient and relatives service, phone conversations, and writings.

- It was difficult to deliver test results and referrals to the right medical doctors.

- The multiple IT systems were slow, not user friendly, and each system required a logon.

- Several medical doctors and nurses were often interrupted in work processes due to supervision of junior doctors or questions related to the IT system.

- Narrow hallways, accumulated goods, and inadequate posting of signs characterized the spatial surroundings.

All in all, the outpatient department seemed to be working, mostly due to a running informal coordination by the staff, and not due to a well-organized system.

\subsection{Codesign workshops}

The workshops with the user group were aimed at developing conceptual ideas for the future organization and functional layout of the new outpatient department. Four workshops, each of a duration of three hours, were accomplished over a period of three months:

Workshop 1: Visions, challenges, and breakdowns

Workshop 2: The dream outpatient department

Workshop 3: Consolidating the dream department

Workshop 4: The department in 3D

At the end the users presented the results from the four workshops for the architect and the department management.

In workshop 1 the users were asked to briefly state, "what characterizes the outpatient department in year 2020?" The main activity then was two sessions in which the users identified breakdowns in the current work practice.

PostIt notes, and flows and sequence models on a large cardboard facilitated the first session. The user group was divided into sub-groups based on their role i.e. doctors, nurses and the secretary. This was done to avid consensus and identify as many break downs as possible. 
In the second session the user group quickly articulated their visions, which encompassed more efficient use of resources, improved support of work processes, more resourceful patients, and improved patient service including same day completion. This was done with the whole user group who were asked to pay attention to the breakdowns when characterizing year 2020.

The main activity at workshop 2 was two design games focusing on sketching the 'dream outpatient department'. The first design game revolved around an A1cardboard with a printed figure of three overlapping ovals (see Figure 1). A number of small cardboard pieces representing different staff members, rooms, and activities had been prepared. The user group was asked to interpret the game board as they liked but they had to design their dream department on the A1 cardboard. The oval shapes did not provide the user group with a resemblance to the layout of their department. This forced the user group to break their established understanding of department layout and rethink.

In the next design game the game board was switched to a rectangular layout, which share natural resemblance to the department layout. The game pieces were rectangular cardboards, and the users were asked to consider the layout of different functions relative to each other. The group was asked to develop the department in this layout. In both design games a LEGO character was introduced as a patient, and the user group has to explain how the patient would pass through a consultation.

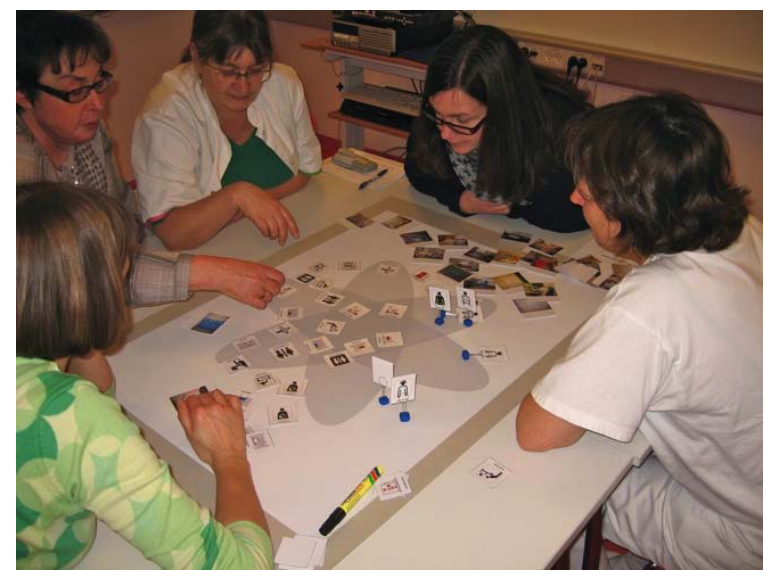

Fig. 1 User group in workshop 2

In both design games the user group developed a new conceptual understanding of the outpatient department. The first design game produced significant results in the sense that the user group was able to develop a radically different department layout. Using the oval shapes the user group quickly explored different designs and began to develop principles for the design. The principles focused both on patient and medical needs. Patients should walk as little as possible and be offered comfortable waiting facilities close to their treatment room. Medical staff should have a back office where patients were not allowed; this should be on a different floor. Patients should be received in the center making it easy to refer them in the right direction. Functions should be grouped according to medical need rather than economic efficiency. The principle was to complete the patient as quickly as possible - which in turn might prove to be economically feasible.

Design game two forced the user group back in the box. However, the user group maintained the principles developed in the first design game and worked hard to create the same solution within this rectangular geometry. This proved very hard as this geometry had many constraints imposed by the classic long hallway connecting all rooms. In the end the user group broke the set geometry to accommodate their focus on a central station for patients and staff (see Figure 2)

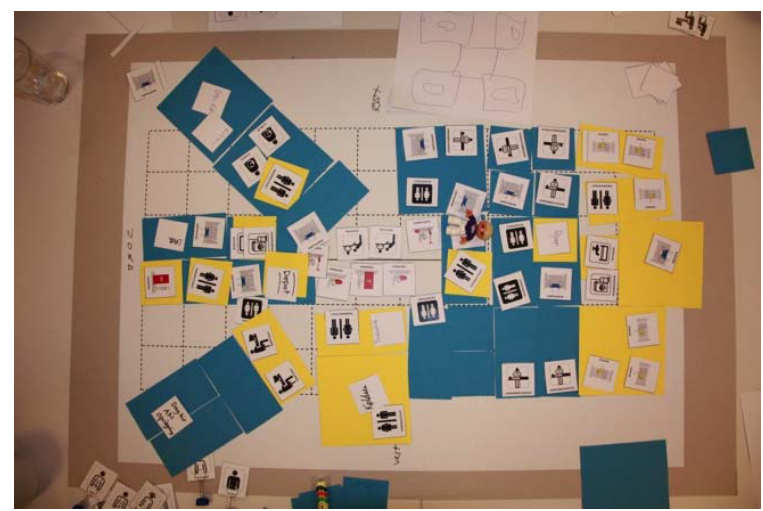

Fig. 2 Design game two in workshop 2

Workshop 3 focused on consolidating the conceptual ideas from the previous workshop. Patient scenarios were introduced and played out on the game board representing the future department. The research team and the users themselves inquired into the concept during the scenarios being played.

During this workshop the new concept for the future department were detailed and things adjusted due to new insights.

Workshop 4 presented the user group with 3D rendering of the researchers perception of the department developed on workshop 2 and 3. The 3D ren- 
derings showed detailed images of waiting area, reception, back office and treatment rooms. The reaction from the user group was a strong focus on the details in the images. Design of the doctor-patient consultation couch, design of the utensil car etc. were immediately discussed. This narrow focus came at the expense of the larger picture - the overall design of the department.

The research team then challenged the users by presenting an idea of a bridge with a captain. This was done because the previous workshops had highlighted that management and organization in the department was less formalized with most initiative placed in the hands of the individual doctors, nurses and secretaries. With no direct management the staff had grown accustomed to make it as they went along. However, the bridge with a captain prompted a discussion that fragmented into two positions: 1) We are better off without management and 2) Coordination would help the many unforeseen situations. The user group left the session with the understanding that a change in physical layout had to be accompanied with a change in organization.

\subsection{Tabletop simulation workshops}

In the third phase selected features of the future department were simulated in three tabletop simulation workshops with the user group.

The first simulation workshop took place around a blank A 0 format cardboard. Other materials included PostIt notes, LEGO characters, felt-tip pens, and most importantly: detailed written patient scenarios for a limited number of patient cases in the outpatient department. The patient scenarios detailed time consumption for each activities that patient would be associated with that patient e.g. 1) patient arriving, 2) reporting to front desk, 3) prepare examination room etc. Two different organizational models were simulated: 1) The patient can be completed during one visit only, and 2) The patient is completed over several visits to the department. The simulation question was: For each model what are the requirements concerning work organization and the spatial layout of the department? The simulation took place by playing the different patient scenarios with LEGO characters representing the patient and the medical staff. Rooms and activities were noted on PostIt's and placed on the game board.

The simulations showed that it would be possible to reduce the number of visits for a cancer patient from five to two, which was a surprise for the user group. This, however, would require a new distribu- tion of roles between the outpatient department and the primary health care sector, as well as improved use of IT between patient and medical staff. In the course of simulation, it was realized that the functional requirements of the examination rooms at the outpatient department was important. The question was weather they should be specialized rooms or multi-functional rooms. Multifunctional rooms would allow for patients to stay in the room during the entire consultation. Otherwise the patient had to move back and forth between a several rooms.

The second simulation workshop focused on two main topics: 1) Multi-functional or specialized rooms and the spatial layout of these and auxiliary rooms, and 2) introducing a bridge and a "captain" aiming at creating an overview and improved coordination. The materials for the workshop were a sort of 3D tabletop simulation using empty shoeboxes as representing rooms, and LEGO characters representing patients and medical staff. The patient scenarios from the first simulation were used again. Members of the user group were playing the role as themselves i.e. doctor, nurse and secretary. The shoeboxes made it easy to play a number of different layouts because they could be rearranged instantly if a new room configuration were to be tested. The main simulation questions were: 1) Is the bridge a physical place or a function? And what type of IT-support is needed? 2) What is the optimal room layout concerning possible bottlenecks and patient waiting times?

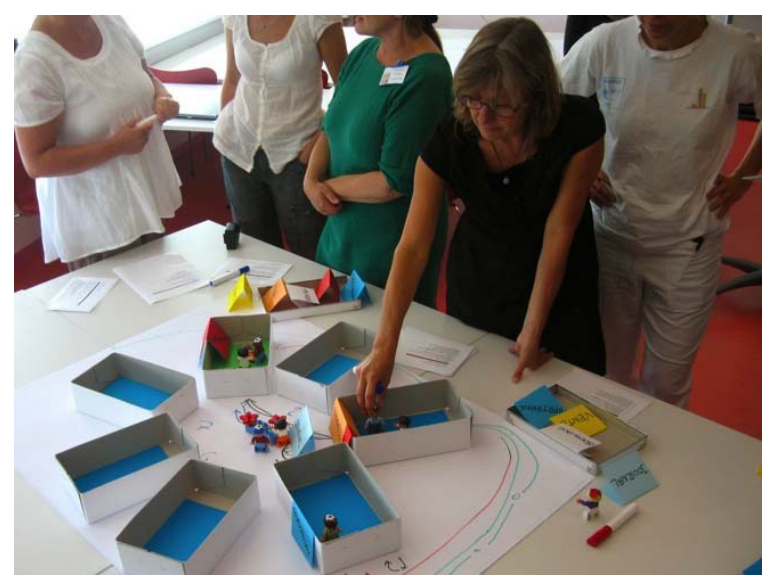

Fig. 3 The star model in simulation workshop 2

The simulations pointed to the multi-functional consultation room as the most adequate (albeit most expensive). Following this realization different configurations of the rooms were simulated. During a break a discussion unfolded and a new configuration model emerged. One of the problems of being the 
captain on the bridge was the limited overview presented by the current long hallway design. It hampered communication and thus coordination.

In a discussion of this problem an idea of a circular layout emerged, which quickly became referred to as the star model (Figure 3). This was a quite new concept for an outpatient department, fully separating a medical staff area in the center, from the patient areas. The shoeboxes were placed in this circular configuration and new simulations of patient scenarios were carried out.

In the third simulation workshop, the star model was consolidated by inviting medical staff from other hospitals to simulate their type of patients in the start model. This provided an indication that the star model could be used in other types of outpatient departments.

\section{Discussion}

In this project the participatory design and codesign approach has been the dominating framework for staging a user-driven innovation process. The user group for this outpatient project has been the medical staff alone. As such, another very important user group, the patients have only been indirectly represented. During the workshops and simulation activities the patients were represented through the medical staff and the research team building on experiences and acting as patients in the simulations. This has clearly been a limitation in the project. It was, however, very difficult to recruit patients from the outpatient department. Even though the researchers and user group tried to act as patients, this perspective was not adequately presented in the project. The consequences were an increased focus on the medical processes surrounding the patient. Patients are important players in healthcare and their views and insights must be used.

Compared to participatory innovation it is clear that the research and user group together did not make up an ideal user-driven innovation team. Expertise within architecture, hospital planning, and interaction design would have made up a more complete team allowing for a dialogue between users and a number of different domain designers.

The outpatient department in this project was scheduled to move to new premises with the design phase beginning roughly at the same time as this project ended. This meant that the user group was in a position to develop and conceptual designing the future outpatient department. This could then have been presented to architects and other relevant designers that were working on the new outpatient departments. Unfortunately the user group was not part of an actual ongoing design project in which they communicated with designers on real design proposals.

The research team tried to compensate this by challenge the user group with questions and suggestions. The lack of architectural and hospital planning experience of the researchers meant that these perspectives were not articulated with sufficient insight and weight.

Given the right knowledge and skills it was possible to challenge the user group into new insights. The most successful example was a research team member, a specialist in patient safety, who introduced the analogy to a ship and the need for a bridge with a captain. This was the first step in the direction of changing the organization of the outpatient department.

Reflecting further on the user-driven innovation process from the research perspective there were some major learning points:

1. The design object was not a product but a complex sociotechnical system entailing elements of culture, politics and learning. The terms of user' acknowledged needs and non-acknowledged needs seem not appropriate in this case as they dissolve in culture and politics in the meeting between different professional domains. The division of labor between medical doctors and nurses turned out to be a sensitive issue. However, when introduced by the researchers in the workshops the staff members were able to articulate their concerns and see new options.

2. The workshops and simulation events can be seen as temporary innovation labs in which experiments with the future design of the department were conducted. Using flexible and design oriented objects in the workshops and simulations seem to enhance innovation and design experiments. The objects were capable of sustaining ideas. The star model developed through and discussion in front of a flipover. The ideas were transferred into drawings and text on the paper screen. Next, they were transferred to the gaming board with shoeboxes, and hence transferred into documents with text and pictures.

3 . The concrete design of the future outpatient department was the major focus. The innovation of the outpatient department as a hospital functionality was by that in the background, maybe resulting in a lower innovation height than could have been reached. On the other side, however, taking departure in the actual 
activities in the department made it easier for the participants to take part in the innovation process.

4. Tabletop simulations seem to be a strong tool to facilitate user-driven innovation. It turned out that even simulation of the current work practice in an outpatient department was an eye opener for innovative ideas. During simulations of possible future configurations new ideas came up, which then could be immediately tested in the simple tabletop simulation.

\section{Conclusion}

This paper has presented experiences from a userdriven innovation process of an outpatient department. The mixing of methods from user-driven innovation and participatory design contributed to develop an innovative concept of the spatial and organizational design of an outpatient department in a hospital. Design games and tabletop simulation seem to be powerful tools in facilitating such a participatory process. However, they are to be planned and configured in the right way by the facilitators. This role could be taken on by a trained ergonomist.

\section{References}

[1] J. Rosted, Brugerdreven innovation - resultater og anbefalinger, Danmarks Erhvervsråd, 2005. (In Danish)

[2] M. Stern, Tensions in human-centred design, CoDesign 7(1) (2011), 45-60. 